\title{
Ultrahigh Vacuum Non-Contact Atomic Force Microscope Observation of Reconstructed Si(110) Surface
}

\author{
Akihira Miyachi*, Hayato Sone and Sumio Hosaka \\ Department of Nano-Material Systems, Graduate School of Engineering, \\ Gunma University, Kiryu, 376-8515, Japan
}

We tried to observe a reconstructed $\mathrm{Si}(110)$ surface using an ultrahigh-vacuum (UHV) non-contact atomic force microscope (NC-AFM). The $\mathrm{Si}(110)$ surface has several characteristic structures, such as the $16 \times 2$, $(17,15,1) 2 \times 1,1 \times 1$, zigzag structures. We succeeded in the AFM observation of the surface formed upon annealing the samples in direct current heating so as to clean the surface. We obtained the same structures in the AFM observation as the proposed $16 \times 2$ model of a Si(110) reconstruction in a STM observation. The UHV NC-AFM results demonstrate that the $\mathrm{Si}(110)$ surface has a characteristic $16 \times 2$ structure for the first time observation. [doi:10.2320/matertrans.47.2595]

(Received July 3, 2006; Accepted August 28, 2006; Published October 15, 2006)

Keywords: ultrahigh-vacuum, non-contact atomic force microscope, Silicon (110), $16 \times 2,(17,15,1) 2 \times 1,32 \times 2$

\section{Introduction}

The scanning tunneling microscope was introduced 23 years ago, ${ }^{1)}$ and many researchers have already examined a wide variety of crystalline surfaces at an atomic resolution. In the semiconductor cleaning process, almost every surface of the low-index planes is reconstituted. As a result, a superstructure is formed on the surface which is different from the atomic arrangement in the bulk crystal. The most well-known superstructure is a $\mathrm{Si}(111) 7 \times 7$, and the DAS model has been proposed for the structure. ${ }^{2}$ Several studies on $\mathrm{Si}(110)$ using low-energy electron diffraction (LEED), reflection high-energy electron diffraction (RHEED) and scanning tunneling microscopes (STM) have been reported. So far, a lot of researchers had to pay their efforts to get atomic images because the $\mathrm{Si}(110)$ plane is very sensitive to impurities. There are many theoretical atomic arrangements, and the sample preparation process is complicated. Yamamoto, et al. have already reported in 1980 that the $\mathrm{Si}(110)$ surface has a characteristic $16 \times 2$ structure, as revealed by RHEED. ${ }^{3)}$ The ideal $\mathrm{Si}(110)$ surface has dangling bonds in the top atomic layer. The top atomic layer of the surface forms a zigzag structure in the [110] direction. A tetrahedron is constructed from unit cells; one side of the tetrahedron is $0.54 \mathrm{~nm}$ long in the [001] direction and the other side is $0.38 \mathrm{~nm}$ long in the [110] direction. A large unit cell is disposed on two symmetrical lines in the $[\overline{1} 1 \overline{2}]$ or the $[1 \overline{1} \overline{2}]$ direction. These lines make a turn-around point at an angle of 70.5 degrees. ${ }^{4)}$ Other clean Si (110) surfaces have a characteristic $1 \times 2,5,6)$ $5 \times 4,^{5-8)} 1 \times 5,^{4-7,9-12)}(17,15,1) 2 \times 1^{6,13-15)}$ or $32 \times 2$ structure. ${ }^{16)}$

In particular, the $(17,15,1) 2 \times 1$ structure is formed on the vicinal plane while the $16 \times 2$ structure is formed on the upper and lower terraces. The step edges are arranged straight along the $[\overline{1} 1 \overline{2}]$ or the $[1 \overline{1} \overline{2}]$ direction. Small terraces appear on each step edge. The terrace width is $2.5 \mathrm{~nm}$ and the step height is $0.19 \mathrm{~nm}$. It has been reported that a phase transition occurs from the $16 \times 2$ structure to the $1 \times 1$ structure at

*Graduate Student, Gunma University
$1043 \mathrm{~K}^{17)}$ The $16 \times 2$ structure of the $\mathrm{Si}(110)$ surface has been investigated by LEED, ${ }^{18)}$ RHEED $^{16)}$ and STM..$^{3,13-15,18)}$ Moreover, Packard., et al. have proposed the $16 \times 2$ structure model for $\mathrm{Si}(110)$ in $1997 .{ }^{19)}$

Although these studies have done with LEED, RHEED or STM, nobody has used AFM for them. We are very interested in observing the reconstructed surface of the $\mathrm{Si}(110)$ using NC-AFM.

In case of the distance between the sample and the cantilever is farther than $1 \mathrm{~nm}$, that interaction force is a Van der Waals force only. In case of the distance between the sample and the cantilever approximate to about $0.8 \mathrm{~nm}$, a covalent bond force acts as the interaction forces. ${ }^{20)}$ In using the NC-AFM with a frequency modulation detection, we can control the distance between the sample and the cantilever by adjusting frequency shifts. In case of the observation of the $\mathrm{Si}$ surface using the Si cantilever, the interaction forces between the surface and the cantilever depends on the distance from the cantilever to the sample.

Usually in case of the observation of a Si surface, the STM observes an electron cloud which is formed from outer electron of $\mathrm{Si}$ atoms; observing the surface equivalent charge density from the Si surface. On the contrary, the NC-AFM observes the physical attractive force or the chemical interaction force generated between the sample and the $\mathrm{Si}$ cantilever. It seems that this physical attractive force is the Van der Waals force, and the chemical interaction force is the covalent bond force, respectively.

In this study, we want to confirm the differences between NC-AFM observations and conventional STM results by adjusting frequency shifts of the Si cantilever of NC-AFM. We first obtained UHV NC-AFM images of a Si(110) surface and then elucidated the characteristic structures of $\mathrm{Si}(110)$, namely the $(17,15,1) 2 \times 1$ step, the $16 \times 2$ structure and the $32 \times 2$ structure. In order to study the superstructure of a reconstructed $\mathrm{Si}(110)$ surface, it is most important to clean the sample surface by annealing it at $900 \mathrm{~K}$. After cleaning the Si surface, we were able to clearly observe the structural change from the meso-crystal structure to the $16 \times 2$ structure. 


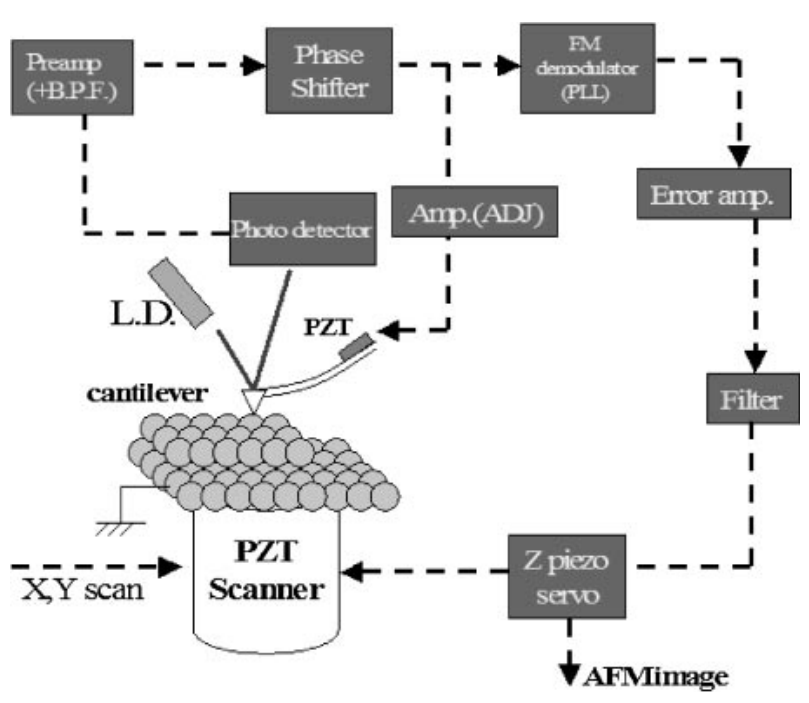

Fig. 1 The block diagram of the NC-AFM system.

\section{Experimental}

In the experiment, we used a UHV NC-AFM system (JEOL, JSPM-4610A), which is schematic design shown in Fig. 1. The base pressure was less than $2 \times 10^{-8} \mathrm{~Pa}$ in the AFM measurement chamber. We have done NC-AFM observation at an attractive force gradient of around $10^{-3}$ $\mathrm{N} / \mathrm{m}$ with the scanning speed of more than $1.0 \mathrm{~s}$ per line. We also used a commercial naked-type conductive $\mathrm{Si}$ cantilever (Mikromash NSC11). The cantilever had a resonance frequency of about $280-310 \mathrm{kHz}$, and the spring constant was about $48 \mathrm{~N} / \mathrm{m}$. The AFM system was equipped with an optical deflected detector and a frequency modulation detector with a phase-locked loop (PLL) for detecting frequency shifts. High-sensitivity detection depends on the $\mathrm{Q}$ factor of the cantilever, and the sensitivity to the atomic force gradient increases as the $\mathrm{Q}$ factor increases. ${ }^{21)} \mathrm{In}$ our system, the Q factor of the cantilever was measured to be about 3000. This value was sufficient to observe this $\mathrm{Si}$ surface with high detection sensitivity.

In the sample treatment, we used an n-type double-sided mirror polished Si (110) wafer (FerroTec Silicon Co.), whose electrical resistivity was $0.5-5 \Omega \cdot \mathrm{cm}$. This Si substrate doped with phosphor. A piece of wafer was cut into $10 \times 2 \mathrm{~mm}^{2}$, and the thickness of the sample was about $0.3 \mathrm{~mm}$. In order to degas the samples, they were heated to a temperature of around $800 \mathrm{~K}$ in an UHV environment for 8 hours by direct current heating. At the first step of the heating treatment so as to clean the surface, the samples were flush-heated three times in a direct current flow at around $1500 \mathrm{~K}$ for $10 \mathrm{~s}$ (total duration: $30 \mathrm{~s}$ ). Then, we subjected the samples to one of three processes.

In the first process, the sample was cooled very quickly from $900 \mathrm{~K}$ to room temperature, which calls "process \#1 sample". This process means that we stopped applying the current for the heating after sample flushing. In the second process, the sample was cooled slowly from $900 \mathrm{~K}$ to room temperature, which calls "process \#2 sample". The applying current for the sample decreased per $0.1 \mathrm{~A}$ from 0.9 to $0 \mathrm{~A}$. And the decreasing span for the sample was 30 seconds per

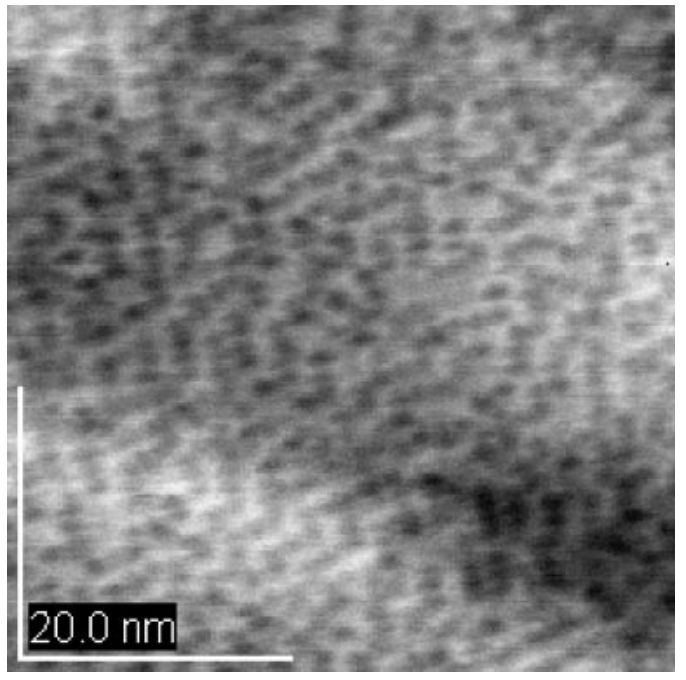

Fig. 2 NC-AFM image of the meso-crystal of Si (110) surface treated by process \#1. Frequency shift was set at $-171 \mathrm{~Hz}$.

$0.1 \mathrm{~A}$. In the $3 \mathrm{rd}$ process, the sample was annealed at a temperature of around $900 \mathrm{~K}$ and kept to anneal it for $3 \mathrm{~min}$. Then, this sample was cooled slowly to room temperature, which calls "process \#3 sample".

\section{Results and Discussion}

\subsection{Quick cooling after flush heating (process \#1 sam- ple)}

Figure 2 shows an NC-AFM image of a $\operatorname{Si}(110)$ surface subjected to process \#1. We took the image at a force gradient of about $51.8 \times 10^{-3} \mathrm{~N} / \mathrm{m}$ (Frequency shift: $-171 \mathrm{~Hz}$ ) and a resonance frequency of about $316 \mathrm{kHz}$. We found only the meso-crystal structures of the $\mathrm{Si}(110)$ surface. We could not see anything discriminative superstructures such as the $16 \times$ 2 or $2 \times 1$. The $\mathrm{Si}(110)$ surface often self-organizes into a $16 \times 2$ reconstruction or surface meso-crystal. The $\mathrm{Si}$ (110) surface cools off rapidly after flushing, the surface does not show a rearrangement structure. We could confirm only the meso-crystal structure without exception in case of the sample rapidly cooling off after flushing in Fig. 2. The meso-crystal is an intermediate state before self-organizing into a superstructure. It is necessary to give a sample annealing time for a longer time. The reason why we cannot confirm the superstructures in the surface is not enough to give the energy for the sample self-organization. It seems that we can observe $16 \times 2$ structure or $2 \times 1$ structure by a longer annealing time. In addition, in order to get a complete clean surface of the sample, we have to study the dependence of annealing time of the sample.

\subsection{Slow cooling after flush heating (process \#2 sample)}

Figure 3(a) shows an NC-AFM image of a $\mathrm{Si}(110)$ surface subjected to process \#2. Figs. 3(b) and (c) correspond to structure model of the Si(110) surface and a cross section profile of a white line in Fig. 3(a), respectively. We took the image at a force gradient of about $19.3 \times 10^{-3} \mathrm{~N} / \mathrm{m}$ (Frequency shift: $-62 \mathrm{~Hz}$ ) and a resonance frequency of about $310 \mathrm{kHz}$. The image shows the superstructure of the 
(a)

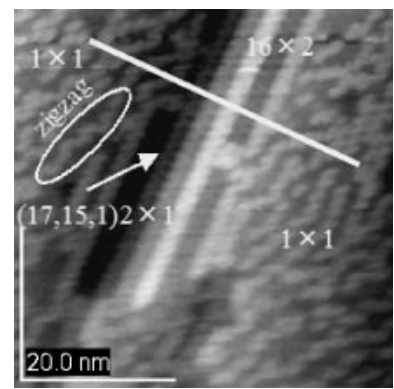

(b)

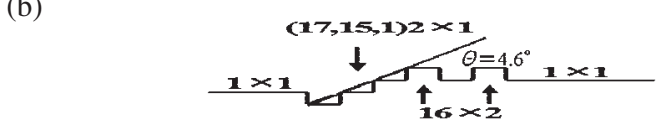

(c)

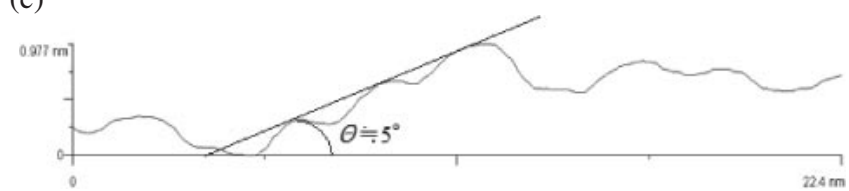

Fig. 3 (a) NC-AFM image of the $(17,15,1) 2 \times 1$ step structure, $16 \times 2$ structure and meso-crystal of $\mathrm{Si}$ (110) surface, (b) a structure model and (c) a cross section profile of a white line in Fig(a). Frequency shift was set at $-62 \mathrm{~Hz}$.

$(17,15,1) 2 \times 1$ step structure and the $16 \times 2$ structure of the $\operatorname{Si}(110)$ surface. Note that the $(17,15,1) 2 \times 1$ structure was observed at the boundary between the vicinal terraces. In addition, the $1 \times 1$ structure and meso-crystal structures were formed in the upper and lower terraces. The step edges were arranged straight along the [112] direction in one stripe or a group of stripes. Our experiment revealed that the annealing period at a temperature of around $900 \mathrm{~K}$ was too short to generate a complete $16 \times 2$ structure. This is because we slowly cooled the samples just after the flush heating. Also, some zigzag structures appeared on the terraces. Although high temperature-STM (HT-STM) reports also showed $16 \times$ $2,(17,15,1) 2 \times 1$ and zigzag structure around $1000 \mathrm{~K}$, a room temperature STM (RT-STM) has not reported yet. ${ }^{17)}$ We succeeded to obtain them by NC-AFM by this slow cooling after flush heating method.

\subsection{Annealing at $900 \mathrm{~K}$ before slow cooling (process \#3 sample)}

Figure 4 shows an AFM image of a Si (110) surface treated by process \#3. We took the image at a force gradient of about $49.2 \times 10^{-3} \mathrm{~N} / \mathrm{m}$ (Frequency shift: $-144 \mathrm{~Hz}$ ) and a resonance frequency of about $281 \mathrm{kHz}$. The characteristic $16 \times 2$ reconstruction is shown in Fig. 4. A sharp-angle corner of about 70.5 degrees is visible. Annealing for $3 \mathrm{~min}$ at around $900 \mathrm{~K}$ proved very effective for rearranging the atoms of the $\mathrm{Si}(110)$ surface to the characteristic $16 \times 2$ structure.

We tried to observe the $\mathrm{Si}(110)$ surface in detail. When we enlarged the corner of the $16 \times 2$ structure to a scanning area of $25 \mathrm{~nm} \times 25 \mathrm{~nm}$, we obtained the atomic structure of the corner, as shown in Fig. 5. We took the image at the same condition as that of Fig. 4 . The $16 \times 2$ unit cell of the $\mathrm{Si}(110)$ surface are indicated by black lines in Fig. 5. The cell has two hexagonal structures, which indicated by white lines. The hexagonal lines run along [ $\overline{1} 1 \overline{2}]$ and [1 $\overline{1} \overline{2}]$ direction. The distances between the hexagonal structures are about $1.3 \mathrm{~nm}$

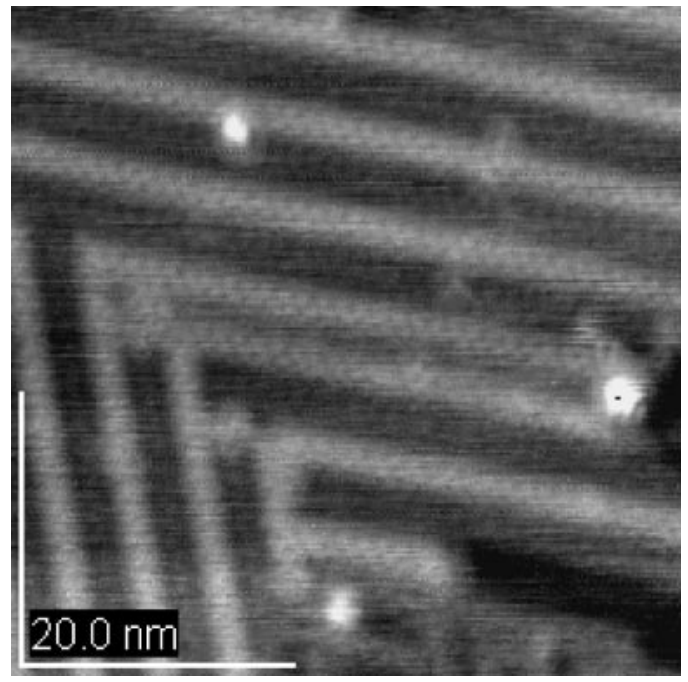

Fig. 4 NC-AFM image of the $16 \times 2$ structure of the Si (110) surface. Frequency shift was set at $-144 \mathrm{~Hz}$.

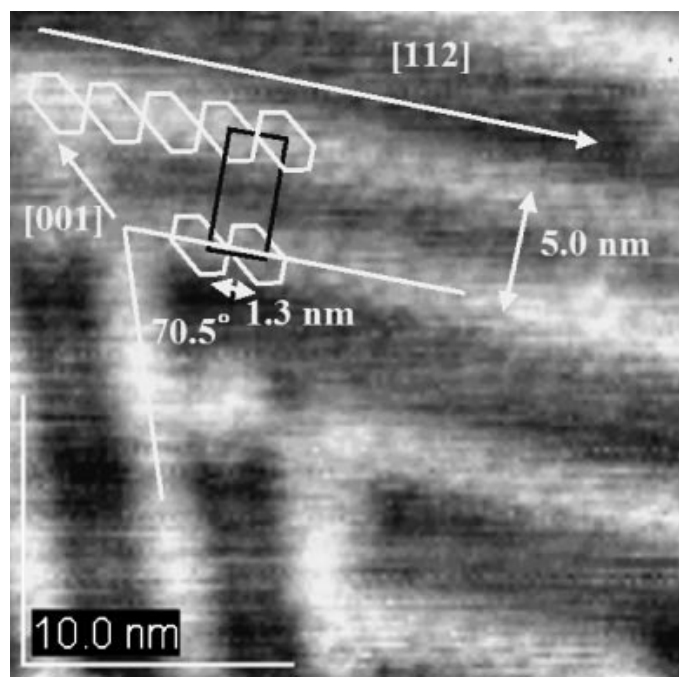

Fig. 5 NC-AFM image of the $16 \times 2$ structure of Si (110) surface. Frequency shift was set at $-144 \mathrm{~Hz}$.

and $5.0 \mathrm{~nm}$. The acute angle formed with the $16 \times 2$ structures in the [001] direction is about 70.5 degrees. The image in Fig. 5 is same as the results of the STM study.

RT-STM can observe only $16 \times 2$ and zigzag structure on Si(110) surface. $^{19)}$ In our AFM observation of $900 \mathrm{~K}$ annealing sample, it seemed that almost every structure showed only $16 \times 2$ structures. So we tried to observe another $16 \times 2$ structure in an other place. As a result, Fig. 6(a) shows the NC-AFM image of $16 \times 2$ structure. We took the image at a force gradient of about $80.6 \times 10^{-3} \mathrm{~N} / \mathrm{m}$ (Frequency shift: $-236 \mathrm{~Hz}$ ) and a resonance frequency of about $281 \mathrm{kHz}$. Secondary, we obtained the characteristic $32 \times 2$ structure, when we took the image at a force gradient of about $82.5 \times 10^{-3} \mathrm{~N} / \mathrm{m}$ (Frequency shift: $-242 \mathrm{~Hz}$ ) as shown in Fig. 6(b).

Although we tried to observe in more detail the $16 \times 2$ structure of the Si (110) surface than in the STM study, atom separation could not be achieved. We think that this structure is similar to the $16 \times 2$ structure. In the case of the $16 \times 2$ 
(a)

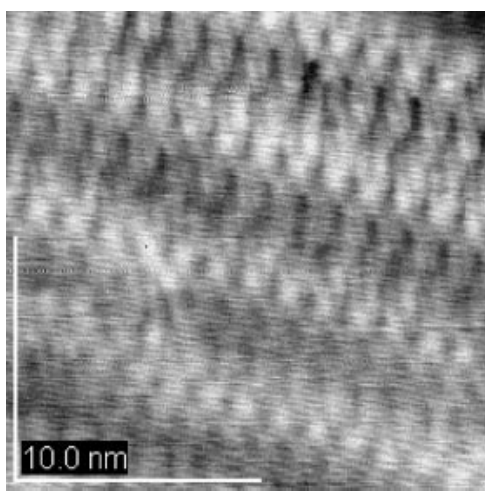

(b)

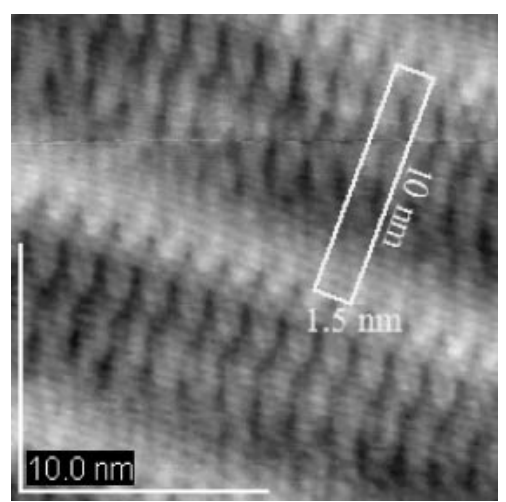

Fig. 6 (a) NC-AFM image of the $16 \times 2$ structure and (b) the $32 \times 2$ structure of the $\mathrm{Si}(110)$ surface. Frequency shift was set at (a) $-236 \mathrm{~Hz}$ and (b) $-242 \mathrm{~Hz}$, respectively.

structure, a pentagon pair at the unoccupied state or a tetragonal pair at the occupied state is formed, and the $16 \times 2$ structures is about $5.0 \mathrm{~nm}$ long. ${ }^{4}$ Usually it is easier to observe long period structures than short-period structures in case of longer distance between the tip and the sample. It was observed the $32 \times 2$ and $16 \times 2$ structure at a little difference place. The $32 \times 2$ structure predicted by an observation result of LEED, however, STM observation is not reported. In our observation, the structure we found was about $10 \mathrm{~nm}$ long, which seems to suggest that it was the $32 \times 2$ structure. We could observe $16 \times 2$ structure when we set a resonance frequency shift with $-236 \mathrm{~Hz}$, and $32 \times 2$ structure was observed when we set it with $-242 \mathrm{~Hz}$. As for this, it seems that the interaction force changed from the van der Waals to the covalent bond force between a cantilever and a sample in the latter case by approaching closer to a sample of a cantilever. The observation condition of $32 \times 2$ structure is about $>1.0 \mathrm{~nm}$ closer than that of $16 \times 2$ structure in the distance between cantilever and the sample. It seems that this point is a threshold in observing $16 \times 2$ structure and $32 \times 2$ structure.

\section{Conclusion}

We tried to observe a reconstructed $\mathrm{Si}(110)$ surface using an UHV NC-AFM. We succeeded in elucidating several characteristic structures of the $\operatorname{Si}(111)$ surface, such as the $16 \times 2,(17,15,1) 2 \times 1,32 \times 2$, zigzag structures. We itemize the conclusions of our NC-AFM observation result.

(1) In the observation of the process of a very quick cooling sample, we observed only the meso-crystal structures.

(2) In the observation of the process of a slow cooling after flush heating, we observed $16 \times 2$, $(17,15,1) 2 \times 1$, $1 \times 1$, meso-crystal and zigzag structures at same time.

(3) The observation of the process of an annealing at $900 \mathrm{~K}$ before slow cooling, we observed only $16 \times 2$ structures. This point is not same as other reported STM observations. We thought $16 \times 2$ structures appeared because zigzag structure grows up in the heating time.

(4) An other area of the observation of the process of an annealing at $900 \mathrm{~K}$ before slow cooling, we observed both the $16 \times 2$ and the $32 \times 2$ structure. In addition, we succeed in the observation of $32 \times 2$ structure by UHV-NC-AFM for the first time.

(5) The case of detecting force of observation is the Van der Waals, NC-AFM observation results are almost same as the reported STM observations. It seems that we can observe almost same result as reported STM observations in setting at a force of the Van der Waals detecting region. On the contrary, it seems that we can observe the $32 \times 2$ structure in setting a force of the covalent bond force detecting region.

We were able to reconfirm the proposed model of the reconstructed $\mathrm{Si}(110)$ structure. This is the first observation of the characteristic structure of the Si (110) surface using an UHV NC-AFM.

\section{REFERENCES}

1) G. Binning, H. Rohrer, Ch. Gerber and E. Weibel: Phys. Rev. Lett. 49 (1982) 57-61.

2) K. Takayanagi, Y. Tanishiro, M. Takahashi and S. Takahashi: J. Vac. Sci. Technol. A3 (1985) 1502-1506.

3) Y. Yamamoto, T. Ichikawa and S. Ino: Proc. of the Annual Meeting of the Physical Society of Japan, Fukui (1980) pp. 300.

4) Toshu An, Masamichi Yoshimura, Izumi Ono and Kazuyuki Ueda: Phys. Rev. B, 61 (2000) 3006-3011.

5) F. Jona: IBM J. Res. Dev. 9 (1965) 375-387.

6) B. Z. Olshanetsky and A. A. Shklyaev: Surf. Sci. 67 (1977) 581-588.

7) H. D. Hagstrum and G. E. Becker: Phys. Rev. B, 8 (1973) 1580-1591.

8) B. A. Nesterenko, A. V. Brovii and A. I. Sorokovykh: Surf. Sci. 171 (1986) 495-500.

9) T. Sakurai and H. D. Hagstrum: J. Vac. Sci. Technol. 13 (1976) 807809.

10) P. Martensson, G. V. Hansson and P. Chiaradia: Phys. Rev. B, 31 (1985) 2581-2583.

11) E. G. Keim, A. van Silhout and Wolterbeek: J. Vac. Sci. Technol. A5 (1987) 1019-1023.

12) R. S. Becker, B. S. Swartzentruber and J. S. Vickers: J. Vac. Sci. Technol. A6 (1988) 472-477.

13) Youiti Yamamoto, Takashi Sueyoshi, Tomoshige Sato and Masashi Iwatsuki: J. Appl. Phys. 75 (1994) 2421-2425.

14) G. Shimaoka: Appl. Surf. Sci. 65/66 (1993) 569-574.

15) Youiti Yamamoto: Phys. Rev. B, 50 (1994) 8534-8538.

16) H. Ampo, S. Miura, K. Kato, Y. Ohkawa and A. Tamura: Phys. Rev. B, 34 (1986) 2329-2335.

17) Youiti Yamamoto, Takashi Sueyoshi, Tomoshige Sato and Masashi Iwatsuki: Surf. Sci. 466 (2000) 183-188.

18) N. Taylor, H. Kim, T. Spila, J. A. Eades, G. Glass, P. Desjadins and J. E. Greene: J. Appl. Phys. 85 (1999) 501-511.

19) William E. Packard and John D. Dow: J. Appl. Phys. 81 (1997) 994996.

20) T. Eguchi and Y. Hasegawa: Phys. Rev. Lett. 89 (2002) 266105-1266105-4.

21) A. Kikukawa, S. Hosaka, Y. Honda and S. Tanaka: Appl. Phys. Lett. 61 (1992) 2607-2609. 